Paper 5 in Per Westerlund's licentiate thesis, KTH Royal Institute of Technology, 2015.

Per Westerlund, Patrik Hilber, and Tommie Lindquist. Condition monitoring of disconnectors in the electric power transmission grid with temperature sensors. Accepted to International Journal of Industrial and Systems Engineering.

OInderscience. Reprinted with permission. 


\title{
Condition monitoring of disconnectors in the electric power transmission grid with temperature sensors
}

\section{Per Westerlund* and Patrik Hilber}

Department of Electromagnetic Engineering, School of Electrical Engineering,

KTH Royal Institute of Technology,

Teknikringen 33, SE-100 44 Stockholm, Sweden

Fax: +46-8-20-52-68

E-mail: per.westerlund@ee.kth.se

E-mail: hilber@kth.se

*Corresponding author

\section{Tommie Lindquist}

Svenska kraftnät,

Swedish National Grid,

Box 1200, SE-172 24 Sundbyberg, Sweden

Fax: +46-8-475-89-50

E-mail: tommie.lindquist@svk.se

\begin{abstract}
This paper presents a method that can be used to rank disconnectors in the electric power system, according to the condition of one of the most critical parts, the contacts. The temperature of the contacts can be continuously measured by a new type of infrared sensor. For the case study, 54 sensors were placed on six disconnectors together with reference sensors measuring the ambient temperature. The measured temperature at each contact is then regressed against the ambient temperature and the current through the disconnector. Both a linear and a quadratic dependency of the current are tested and the quadratic one explains the data best. The coefficient of the current serves as a measure of the condition of the contact. Then, it becomes possible to identify in which contacts the resistance has increased the most. As a consequence, it is possible to make better maintenance decisions for the disconnectors.
\end{abstract}

Keywords: electric power transmission; disconnectors; electric contacts; temperature measurement; infrared sensors; reliability; condition monitoring.

Reference to this paper should be made as follows: Westerlund, P., Hilber, P. and Lindquist, T. ( $\mathrm{xxxx}$ ) 'Condition monitoring of disconnectors in the electric power transmission grid with temperature sensors', Int. J. Industrial and Systems Engineering, Vol. X, No. Y, pp.xxx-Xxx.

Biographical notes: Per Westerlund received his MSc in Electrical Engineering from KTH Royal Institute of Technology, Stockholm, Sweden, in 1996 and is currently a PhD student there within the field of reliability of the electrical power system. He has also worked with radio communications. 
Patrik Hilber received his MSc in Systems Engineering and the Tech. Lic. and $\mathrm{PhD}$ in Electric Power Systems from KTH Royal Institute of Technology in 2000, 2005, and 2008, respectively. Since 2008, he has been the Research Leader for the RCAM Research Group, working as a researcher and Assistant Professor at the School of Electrical Engineering, KTH. Other responsibilities involve being the Deputy Director of the Swedish Research Centre SweGRIDS and work in two educational boards. His research interests include maintenance optimisation and reliability modelling of power systems.

Tommie Lindquist received his PhD in Electrical Engineering from KTH Royal Institute of Technology, Stockholm, Sweden in 2008. His research focused on the reliability modelling of electric power equipment with special regard to the effect of maintenance. He is currently with Svenska kraftnät (Swedish National Grid), Stockholm, Sweden. His main focus at Svenska kraftnät is the technical asset management of high-voltage switchgear and instrument transformers. This includes evaluating and developing maintenance strategies as well as reliability monitoring and managing technical specifications.

This paper is a revised and expanded version of a paper entitled 'Condition monitoring of the disconnectors in the electric power transmission grid with temperature sensors' presented at Maintenance Performance Measurement and Management (MPMM 2013), Lappeenranta, 12-13 September 2013.

\section{Introduction}

\subsection{Background}

The electric power transmission grid consists of the overhead lines and cables connecting generators and substations. It transmits electric power across large distances with the highest voltages in the electric grid. Therefore, it has to be reliable and it has an availability of at least $99.999 \%$ in Sweden. To achieve such a high availability, the number of planned outages for maintenance must be reduced, but at the same time the equipment should be in a good condition to avoid outages due to failure. In the two most northern electric areas of Sweden, where a large amount of hydropower is produced, it is challenging to plan outages in order to carry out maintenance or to connect new equipment, since the network capacity is nearly fully utilised. This problem is expected to increase even further in the near future [Svenska kraftnät, (2013), pp.61-67].

Two major blackouts in 1983 and 2003 in Sweden were caused by overheated disconnector contacts [Andersson et al., (2005), p.1984; Eriksson, 2005]. The disconnectors are found at substations and they are opened during maintenance in order to isolate other equipment from high voltages. Thus the disconnectors contain electric contacts, whose resistance can increase due to degradation. When the resistance is too high, too much power is dissipated, which can lead to damage in the material and eventually to a failure. In order to prevent failures and to plan the maintenance, the resistance of the contacts can be monitored and used as a condition measure of the disconnector. There is a relatively long chain of events and analysis needed to make rational decisions about maintenance. We call this chain the reliability chain and it is illustrated in Figure 1. 
Figure 1 The reliability chain

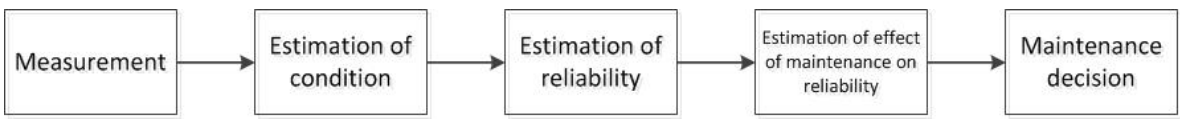

One possibility to measure the resistance, $R$, of an electric contact is to use the definition, that is, the voltage $V$ divided by the current $I$, a variant of Ohm's law, $R=V / I$. Stanisic (2011) presents an equipment to measure the resistance according to this method. The problem is that the disconnector has to be completely without voltage, which normally means that the whole line to the substation needs to be disconnected at the other end. As it will decrease the transmission capacity of the grid, it can only be done with a long time interval such as every five years or more seldom. Also the voltages used at that kind of measurement are quite low, so the measured resistance might differ from the actual resistance at the operating voltage of the disconnector. There are also instruments that can measure the voltage drop across a contact with live equipment, but they do not withstand the high voltages in the transmission network.

Another possibility is to measure the resistance indirectly, for example as an increase of temperature. It can be done with temperature sensors or by thermography, i.e., using a camera that captures infrared radiation. It has been shown that the correlation between the temperature rise and the resistance is around 0.9 (Braunovic et al., 2009), so any of these parameters can be utilised. Braunovic et al. (2005) have deduced formulas that express how the contact resistance increases with time due to oxidation. The formulas predict sometimes a sharp increase of the resistances a function of time, sometimes just a linear growth, depending on the distribution of contact spots between the two conductors. The formulas are based on the theories of the physics of electrical contacts presented in Holm (1967). Muhr et al. (2006) studied experimentally the temperature of contact contacts with varying currents and wind speeds using thermography.

Jadin and Taib (2012) provided a review of thermography for diagnosing electrical equipment. They conclude that thermography is useful for detecting hot spots, but that it requires skill to interpret the images. Bagavathiappan et al. (2013) have made another review of thermography that covers different technical areas, with a list of 20 articles in electrical engineering. Now new computer-based programs have been developed which make the monitoring faster and do not require skilled staff. For example, Huda and Taib (2013) described a method to detect if a device is defective or not based on a thermographical image, which makes the interpretation of the images easier, although this method still requires some manual work to indicate which part of the image the program should process. With further development, it should be possible to automatise the image recognition. Korendo and Florkowski (2001) presented a toolbox for analysing infrared images, which also is able to detect trends. Still it is necessary to preselect the regions to be analysed.

As thermography normally requires that a technician visits the substation, it is not uncommon to make the measurements when the current is low, which gives a low accuracy according to Lindquist et al. (2005). In addition, it is difficult to get an absolute temperature value, since the camera should be used at the same spot and with the same angle to get repeatable measurements. Consequently, thermography is good for comparison between different parts of a substation at a given time. A strategy for 
inspection with thermography has been developed earlier by Lindquist and Bertling (2007).

Since the measurements with thermography are not easily repeatable nor carried out frequently, a new method to measure the temperature has been developed. There are descriptions of systems with temperature sensors (Zhang et al., 2012; Huang et al., 2011). Both papers tell mostly about the structure of the system and the wireless transmission of data. Their sensors are conventional thermistors, so they measure the temperature as a change in resistance in a semiconductor, and the sensors are powered by induction. Shi et al. (2002) use a thermopile, which is an advantage since it directly compares the temperature of the object to be monitored with the ambient temperature. These papers do not explain how the monitored data should be interpreted.

It is possible to inspect the time series of the temperature in different parts of an electrical device and deduce the cause of a temperature rise as shown by Chudnovsky (2007). As a next step, that paper shows that a higher slope between temperature rise and current indicates a defective contact, when calculating the linear regression monthly. There are illustrative examples of temperature curves and their interpretation. For example, Livshitz et al. (2005) describe in detail both the measurement system and the presentation of the data in a monitoring system, which shows trends and creates alarms. Livshitz et al. (2005) also test models to explain the temperature rise with the current. They find that a quadratic dependency on current is better than a linear one. Chudnovsky et al. (2008) present a regression model with an additional factor in the coefficient of the squared current that expresses the aging of contacts. This factor causes an increase of the temperature. They show also that it is important to measure near the contacts.

Since IR sensors have become cheaper and they have a long battery life due to low power consumption, they are a good complement to thermography since they measure the temperature more often and thus they can get measurements with higher loads than with thermography. Furthermore, there is no difference in position or in angle between different measurements, since they are fixed, and there is no extra layer of image processing, as the contacts only look at the same point and they are cheap enough to be placed at many points. The drawback is that the IR sensors only measure at specific points, which means that knowledge about the equipment becomes important, as unexpected fault indicators might be overlooked (Lindquist, 2011). Thus a system with sensors at specific points and thermography complement each other.

\subsection{Outline of the paper}

Thermography is not suitable for continuous monitoring of temperature at specific points in the equipment. A system with IR sensor is more suitable due to the reasons presented earlier. The papers by Zhang et al. (2012), Huang et al. (2011) and Shi et al. (2002) describe systems with conventional sensors, but they do not explain how the monitored data should be interpreted, whereas Chudnovsky (2007), Chudnovsky et al. (2008) and Livshitz et al. (2005) have illustrative examples of temperature data and both a linear and a quadratic model to explain the data. However, they study medium-voltage circuit breakers, with sensors in closed compartments, while this article studies sensors on disconnectors, which are outdoors, so the thermal flow is different. Also circuit breakers have longer time constants for the heat transfer than disconnectors. Hence the motivation for this paper is to develop models to explain the temperature values of sensors placed at the contacts of disconnectors. The sensors use infrared technology, which is a novelty. 
As mentioned previously, the electric grid gets more heavily loaded, and it becomes more difficult to plan an outage in order to maintain a disconnector. Therefore it is valuable to monitor disconnectors in order to plan their maintenance more carefully. In addition, the measurements provide data for improved aging models of disconnectors. Thus the maintenance plan, even for the disconnectors that are not monitored, could be made more accurate.

In order to make good maintenance decisions, it is necessary to advance along the reliability chain in Figure 1. The purpose of this study is to cover the first two steps of the reliability chain. The measurement system converts the actual temperatures to numbers, which in the second step are used to estimate the condition of the contacts in the disconnector. The condition can be used to sort the disconnectors according to the need for maintenance.

Section 2 includes a short description of the electrical system in order to explain where the disconnectors are installed and the function of the disconnector. Section 3 introduces the measurements and the preliminary data treatment. The modelling is presented in Section 4, with three different models. Finally the results are discussed and future work is presented in Section 5.

Figure 2 A schematic view of the electric grid (see online version for colours)

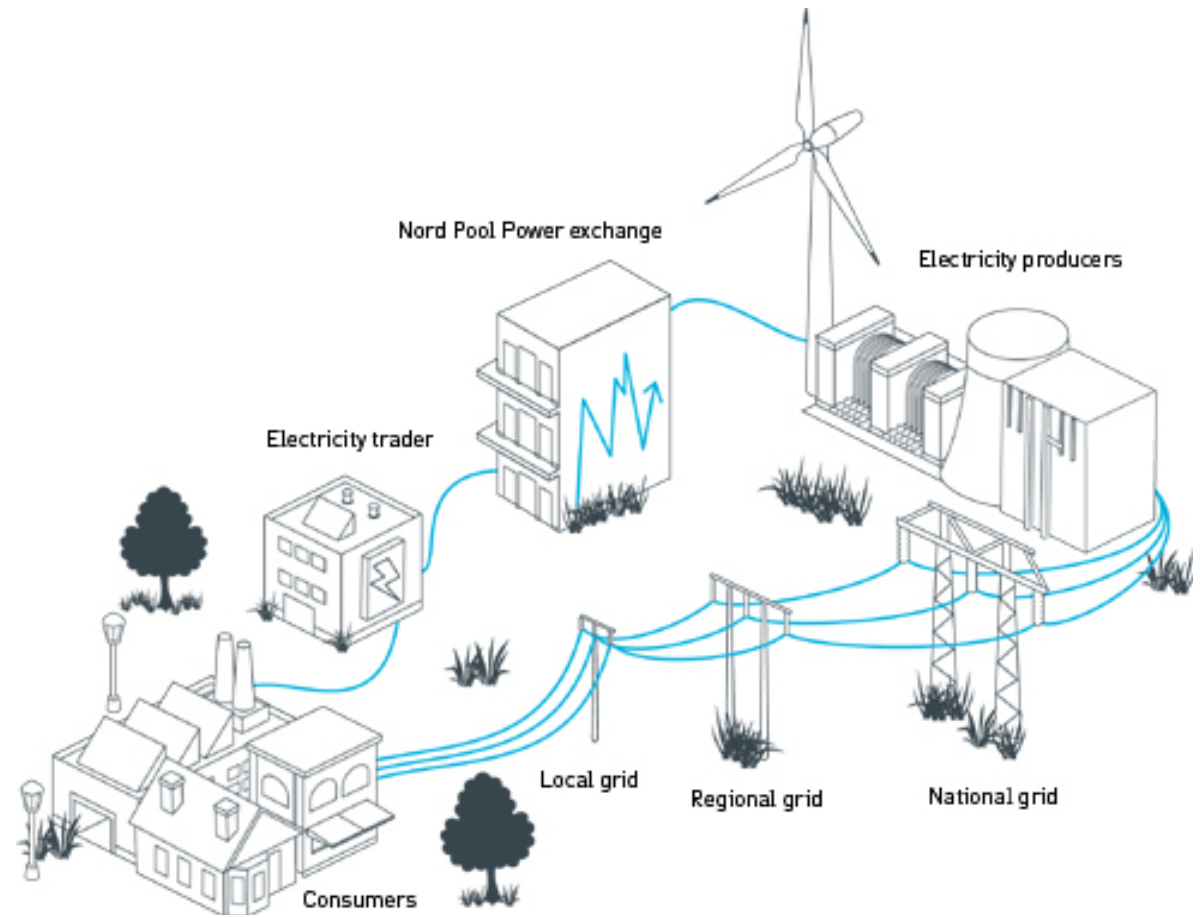

Source: Reproduced with permission from Svenska kraftnät 


\section{The electrical system as an application}

The section gives some background information on the electric transmission system and its components, as the disconnectors. In Sweden, the electric grid is divided in three parts: national grid, regional grid and local grid, as illustrated schematically in Figure 2. Sometimes, the terms electric power transmission network and electric power distribution network are used. The flow of electric power is from the right to the left in the lower part with decreasing voltages and, as a consequence, decreasing length of the poles. The connection in the upper part between the producer and the consumer represents economic transactions.

\subsection{Electric transmission system}

The electric transmission system consists of overhead lines and, occasionally, cables that transmit electric energy across a country, with interconnections to other countries. The lines connect at substations, which serve to lower the voltage and to distribute electric power. The transmission system is in general meshed, which means that there are several paths between two points, and it uses the highest voltages, in Sweden $400 \mathrm{kV}$ and $220 \mathrm{kV}$ (kilovolts). Figure 3 shows the Swedish transmission system, which consists of $15,000 \mathrm{~km}$ of lines and 150 substations (Svenska kraftnät, 2014). The transmission system is divided into four bidding areas (elområde in Swedish) based on the bottlenecks in transmission capacity.

\subsection{Equipment at the substations}

In the substations there are transformers, which lower the voltages, for example from $400 \mathrm{kV}$ to $220 \mathrm{kV}$. There are also switches, which are of two different types: disconnectors, also known as disconnect switches or isolator switches, and circuit breakers. The purpose of the disconnector is safety, to isolate certain equipment from high voltages and it is not designed to break currents. The circuit breaker is the actual breaker of the current.

The studied disconnectors are so called centre-break and they consist of two arms, which can rotate around a ceramic insulator. The ends that are not connected to the insulators join each other when it is closed. Figure 4 shows how the disconnector is constructed. Most of the time the disconnector is closed and carries current and it is only opened a few times every decade, during maintenance of other equipment.

In order to prevent failures in the disconnectors, the possible causes should be studied. The resistance can increase in the contacts points, both in the main contact between the two arms and in the contacts between the moving arms and the connecting cables. A high resistance causes high power losses according to Joule's law ( $P=R \cdot I^{2}$ where $P$ is the power, $R$ the resistance and $I$ the current). With high power losses, the temperature of the conductor will rise, which could cause damages in the material. Consequently, the contact resistance could be regarded as a condition to monitor. 
Figure 3 The Swedish electric transmission system with the $400 \mathrm{kV}$ lines in red and the $220 \mathrm{kV}$ lines in green (see online version for colours)

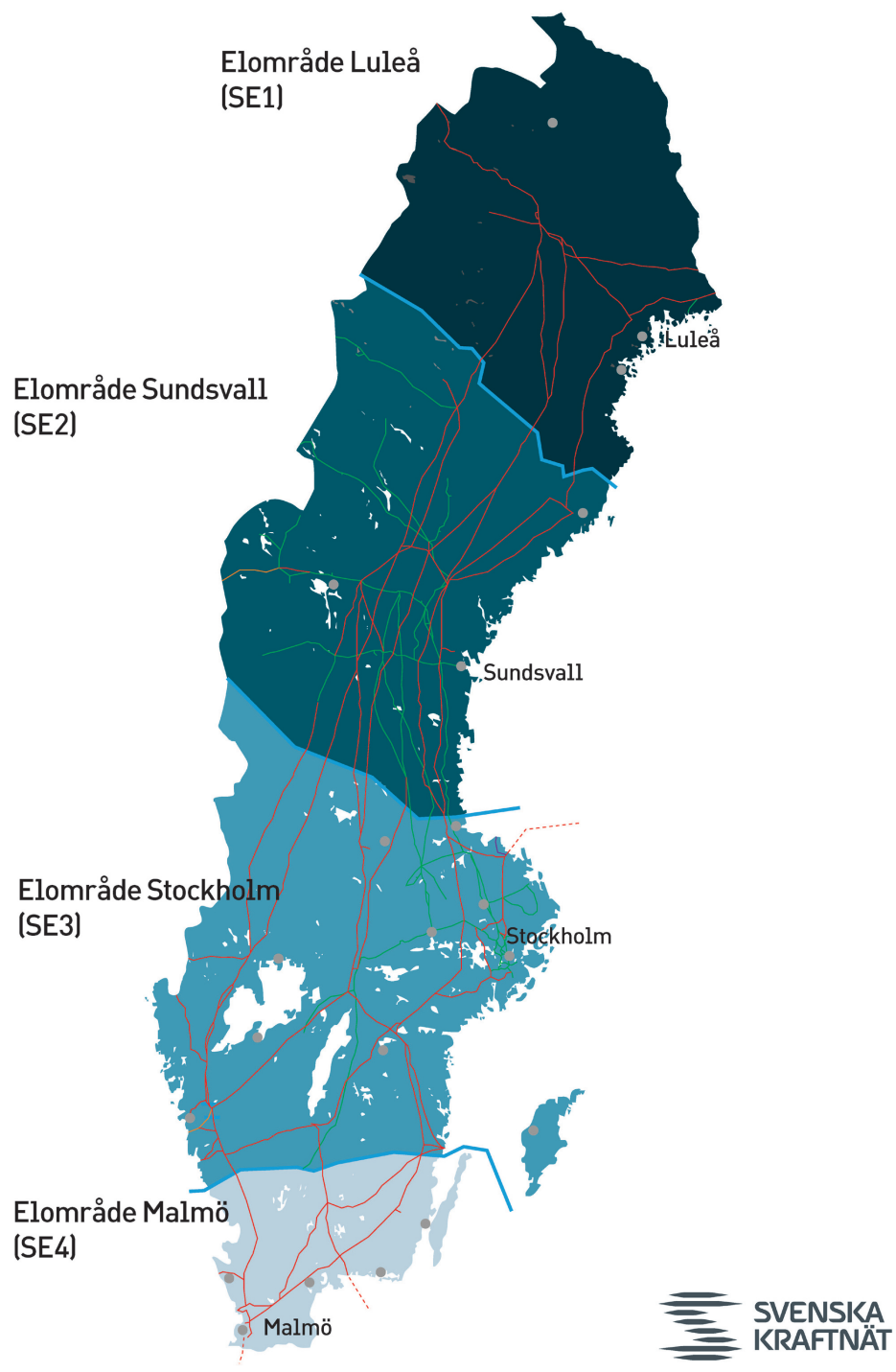

Source: Reproduced with permission from Svenska kraftnät 
Figure 4 Example of a disconnector with the arms joined at the bulky middle part (see online version for colours)

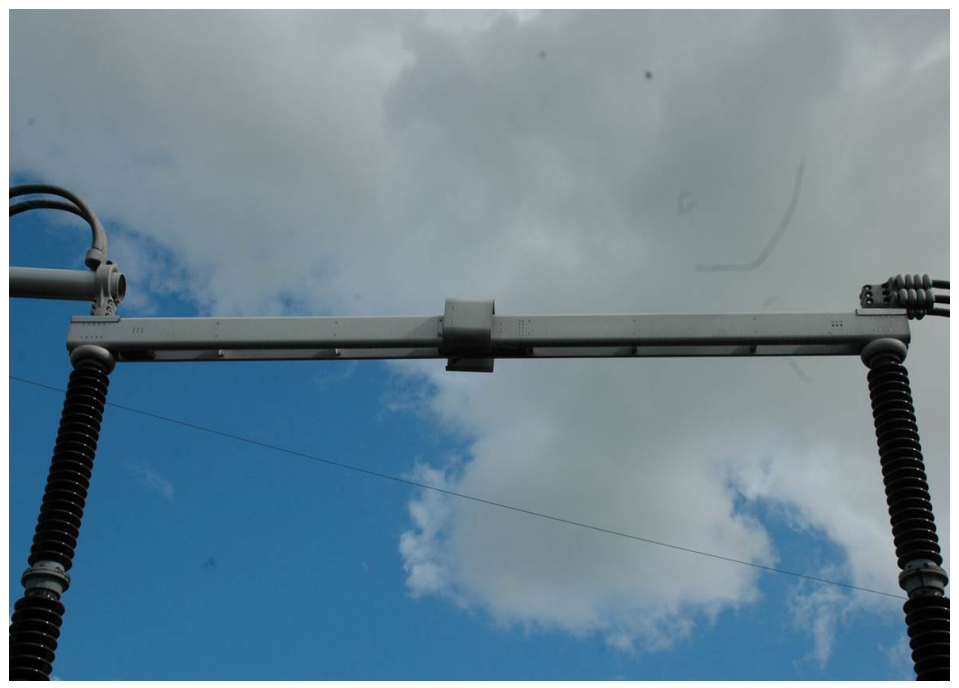

Source: Svenska kraftnät

Figure 5 Example of a disconnector with a sensor placed on the arm above the insulator as shown by the arrow (see online version for colours)

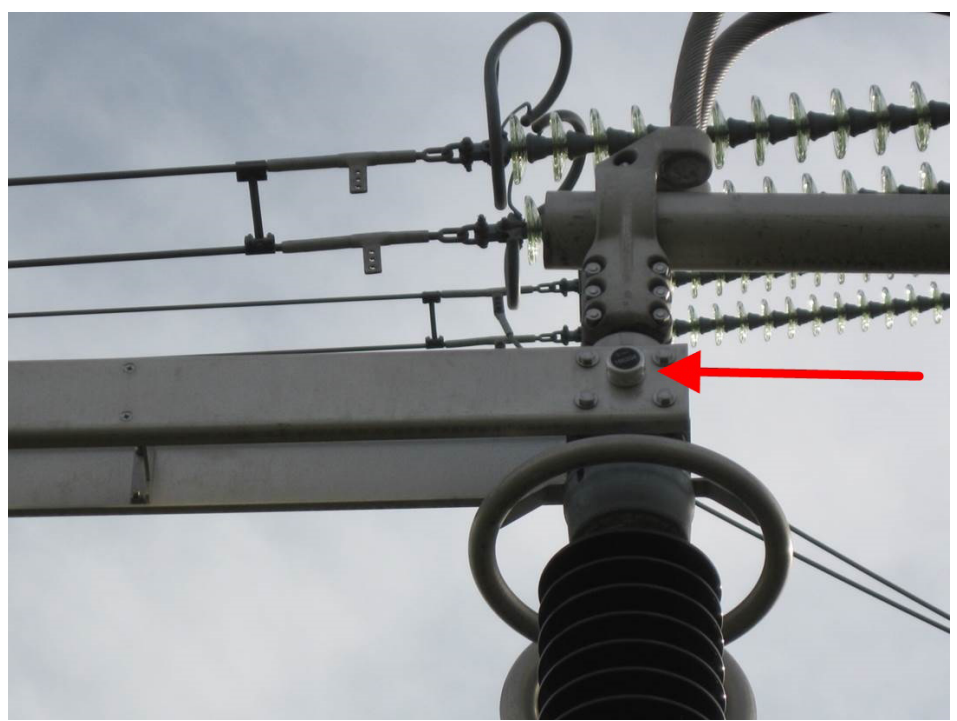

Source: Svenska kraftnät 
Figure 6 Placement of the sensors (small squares) on a disconnector, three on each phase and a reference sensor close to the ground

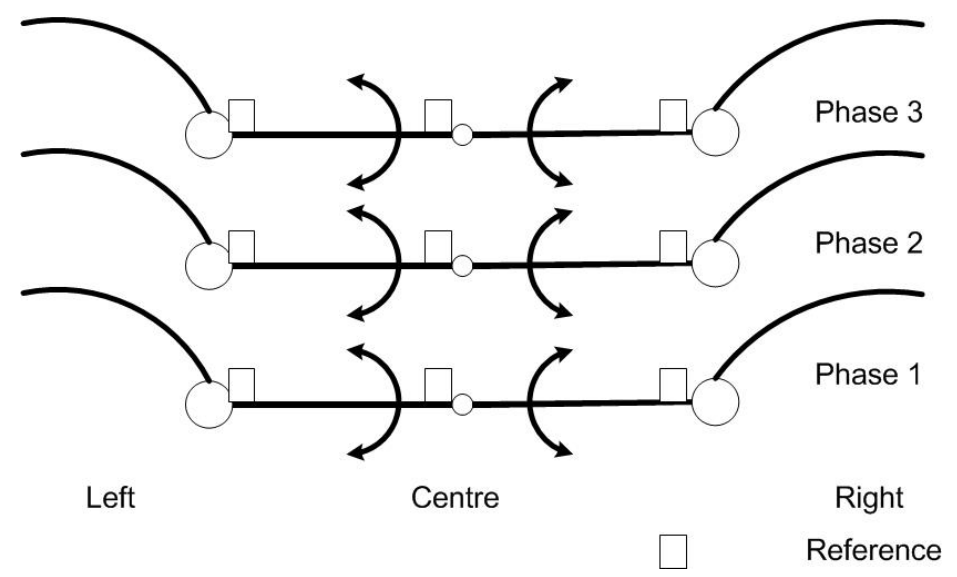

\section{Measurements and preliminary data treatment}

The objects of this study are six $400 \mathrm{kV}$ disconnectors at two substations in Sweden. The disconnectors were chosen since they represent a stratified sample of the population, as the disconnectors are from three different decades: 1970s, 1980s and 1990s. Nine temperature sensors have been placed on each for the project. There are three sensors along each of the three phases, two at the contacts between the two moving arms and the respective support and one at the contact between the arms as seen in Figures 5 and 6 . There are also reference sensors at each substation in order to record the ambient temperature. The temperature is transmitted every eight minutes to a base station at the substation by radio, so there are no cables to the sensors, which is necessary since the sensors are placed on surfaces that are at high voltages. An insulated pole has to be used to place them. The sensors have a battery with a design life of ten years. The accuracy of the temperature sensors is \pm 0.5 degrees centigrade.

The data has to be cleaned from unjustifiable values, mostly values near the absolute zero, which are due to error in the measurement sensors. Also there are time intervals, where there is no data, due to some communication problems. Figure 7 shows data from the sensor at the centre contact of phase 1 of the three phases for one of the disconnectors. The two empty intervals around 60 and 100 days after the start of the measurements are due to a malfunction of the data collecting system.

The distribution of the temperature at one contact is shown in Figure 8, which has a mean of $0.48^{\circ} \mathrm{C}$ and a variance of $35.7\left({ }^{\circ} \mathrm{C}\right)^{2}$, which gives a standard deviation of $5.97^{\circ} \mathrm{C}$. The variation is quite large around the mean. In order to understand this variation, the temperature at a contact is compared with the reference sensor, which is not heated by the current that goes through the disconnector. A problem when making this comparison is that some sensors transmitted data 15 times as often as the others. Furthermore, the data has to be interpolated since the sensors are not synchronised in time. The interpolation is linear and an example result is shown in Figure 9 where there is 
a temperature drop during three winter days in December 2011. There is a difference between the two sensors of less than ten degrees, which will be explained in the following sections.

Figure 7 Example of time series of the temperature

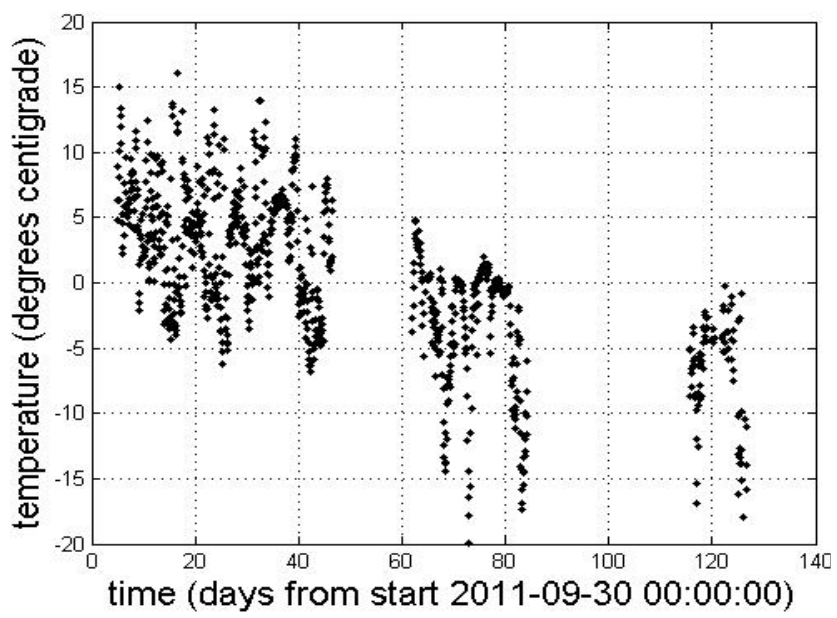

Note: The dots are from the sensor at the centre contact of phase 1.

Figure 8 Histogram of the temperatures of the centre contact of phase 1 (see online version for colours)

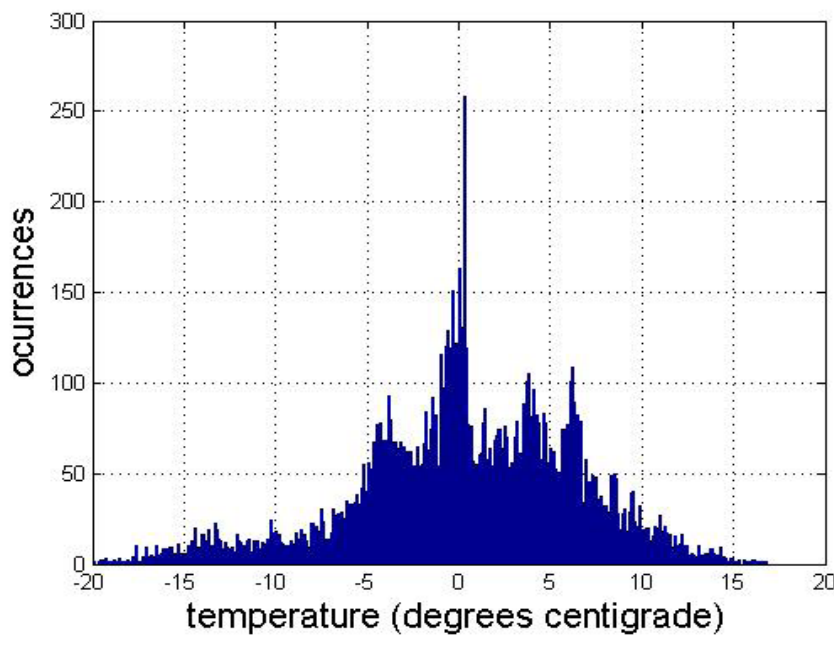


Figure 9 Time series of the temperature at two sensors

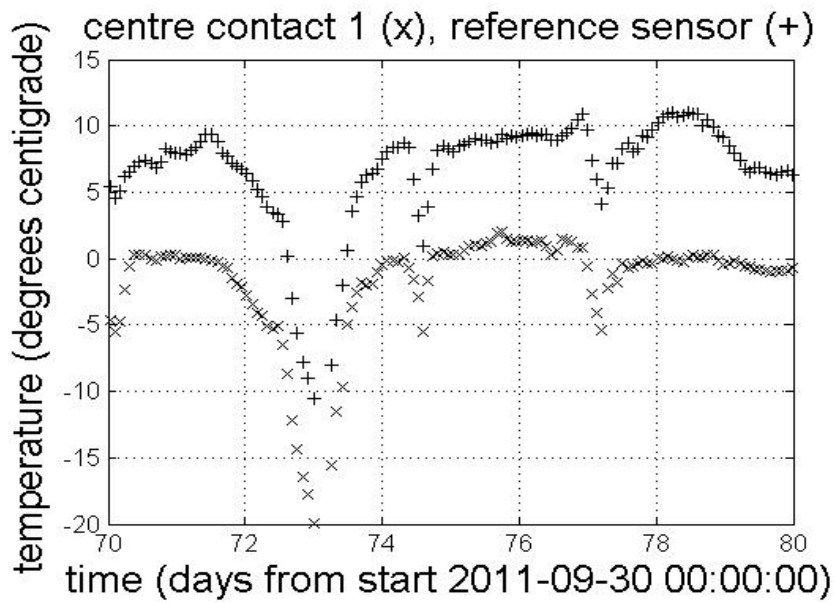

\section{Modelling of the data}

In this section three models are presented to explain the temperature variation of the contacts. The first model includes only the temperature of the reference sensor, whereas the other two include also the current, linearly and quadratically.

\subsection{A basic model}

In order to get a relation between the temperature of two sensors, the time series have been plotted against each other as in Figure 10. In all the figures the data comes from two sensors on the same disconnector, one is called phase 1 centre and it is placed at the contact between the arms, the other one is called reference and it measures the ambient temperature. A basic first step is to make a linear regression, i.e., to fit a equation:

$$
T_{2}=b_{0}+b_{1} \cdot T_{1}
$$

where $T_{2}$ and $T_{1}$ are the temperatures at the two sensors at the same time and $b_{0}$ and $b_{1}$ are two parameters to be determined. The result is shown in Figure 10.

The linear regression is a way to explain the variation of one sensor with the variation of the other. Since there are more than two temperature pairs, let it be $n$, the system of equations is over-determined and the solution is given by a least squares algorithm. That is, the equation system to be solved is:

$$
T_{2 i}=b_{0}+b_{1} \cdot T_{1 i}+\epsilon_{i}
$$

for $i$ going from 1 to $n$ and the sum of all $\epsilon_{i}^{2}$ should be minimised [Hogg and Tanis, (1993), pp.488-509]. The $\epsilon_{i}$ is called residual for each pair and represents what could not be explained by formula (1). The results from one disconnector are shown in Table 1. The sensors at the contacts are regressed against the reference sensor. 
Figure 10 Scatter plot of two time series of temperatures

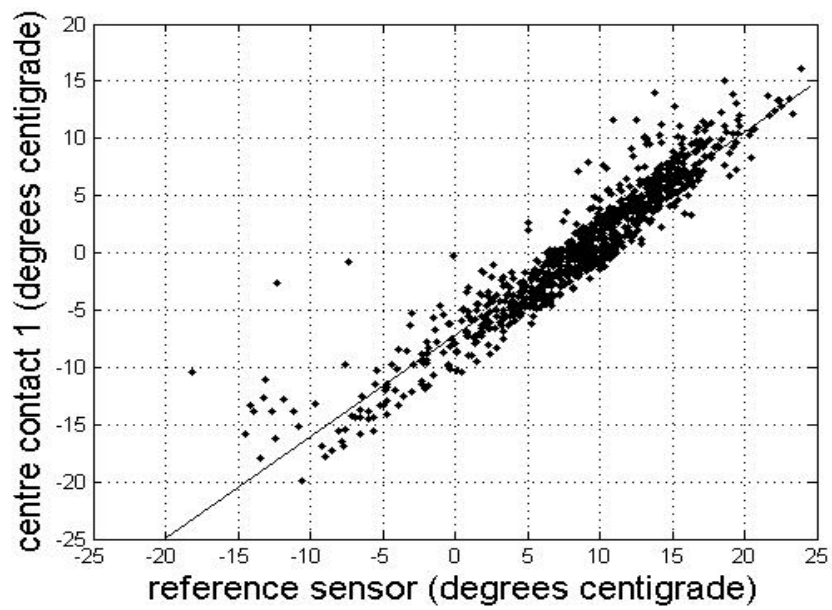

Note: The line comes from the regression of the phase 1 centre sensor against the reference sensor, $T_{2}$ respective $T_{1}$ in the formula (1)

Table 1 Parameters and $R^{2}$ estimated according to formula (1)

\begin{tabular}{lccc}
\hline Sensor & $b_{0}\left({ }^{\circ} \mathrm{C}\right)$ & $b_{1}$ & $R^{2}$ \\
\hline Phase 1 right & -6.89 & 0.930 & 0.949 \\
Phase 1 centre & -7.18 & 0.887 & 0.887 \\
Phase 1 left & -6.14 & 0.915 & 0.924 \\
Phase 2 right & -6.57 & 0.904 & 0.941 \\
Phase 2 centre & -6.54 & 0.911 & 0.935 \\
Phase 2 left & -6.86 & 0.926 & 0.944 \\
Phase 3 right & -7.52 & 0.954 & 0.926 \\
Phase 3 centre & -7.64 & 0.940 & 0.941 \\
Phase 3 left & -5.41 & 0.886 & 0.900 \\
\hline
\end{tabular}

It is important to study the residuals to find out how successful the regression was in explaining the variation of $T_{2}$. Figure 11 shows the histogram of the residuals from the regression of phase 1 centre against the reference sensor. The variance of the residuals is $4.04\left({ }^{\circ} \mathrm{C}\right)^{2}$. In order to investigate how much of the behaviour of $T_{2}$ is explained by this model, it is useful to divide the variance of the residuals by the variance of $T_{2}$, in this case phase 1 centre, and then subtract the result from 1 . This measure is called $R^{2}$ and amounts to 0.89 , which is quite good, since it lies always between 0 and 1 and the closer $R^{2}$ is to 1 , the better is the model [Sen and Srivastava, (1990), p.14]. 
Figure 11 Histogram of temperature residuals (see online version for colours)

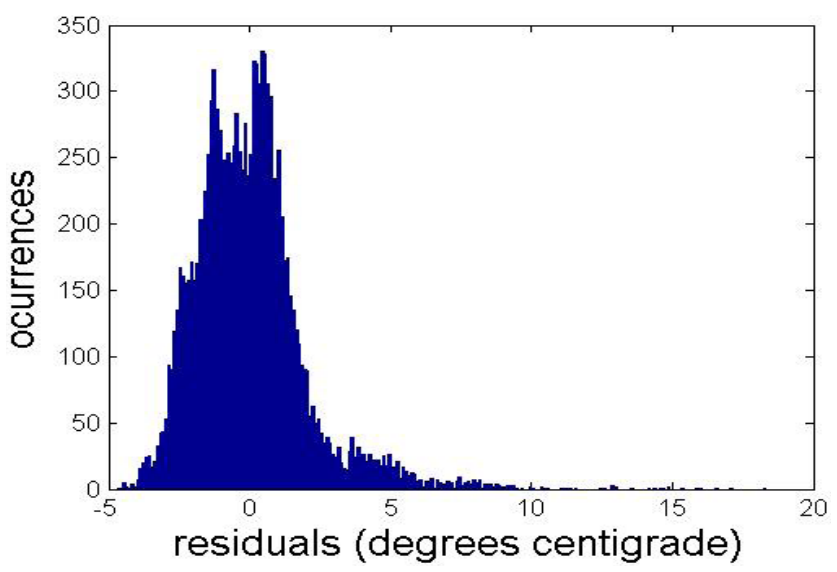

There are other measures that characterise the distribution of the residuals as the skewness, which is 2.22. This means that the right tail is bigger than the left tail.. A symmetrical distribution has a skewness of 0 . The kurtosis or excess is 14.3 , which means that the distribution is much taller than the normal distribution around its mean. The skewness is calculated as the sum of the third power of the deviations from the mean and the kurtosis as the fourth power. Both are normalised by the standard deviation to a suitable power, so that they become dimensionless [Cramér, (1945), pp.183-184]. The temperature data of phase 1 centre has a skewness of -0.46 and a kurtosis of 3.35 , which is close to 3 , the kurtosis of the normal distribution. Thus the distribution of the residuals differs more from the normal distribution than the distribution of the temperature values. This reveals that there should be some more factors to investigate.

The sensors at the contacts are about seven or eight degrees colder than the reference sensor according to the coefficient $b_{0}$. The reason for this is that the reference sensor is placed on the driving mechanism, which is heated. When the reference sensor gets warmer, the temperature of the contacts increases with about the same amount, which is the same that $b_{1}$ is nearly 1 . As the purpose is to rank the different contacts according to their condition, it is valuable to estimate the variation of the parameters in relation to their value. The measure is the standard deviation divided by the mean, which is -0.103 for $b_{0}$ and 0.0251 for $b_{1}$. This model explains between 0.89 and 0.95 of the variation in the sensors at the contacts, which is seen as a good result.

\subsection{A linear model}

Although the basic model, formula (1), explained a great amount of the variation in the data, it was shown in the second last paragraph of the preceding subsection that there are something unexplained in the variation of the temperature. A factor to be accounted for is the current, which is shown in Figure 12. The values of the current are given as an hourly average. Hence, these values need to be interpolated in order to facilitate the regression with the temperature values measured every eight minutes. The interpolation is linear. The temperature residuals are plotted against the current to investigate the influence of the current on the temperature. 
Figure 12 Variation of the current in one of the disconnectors
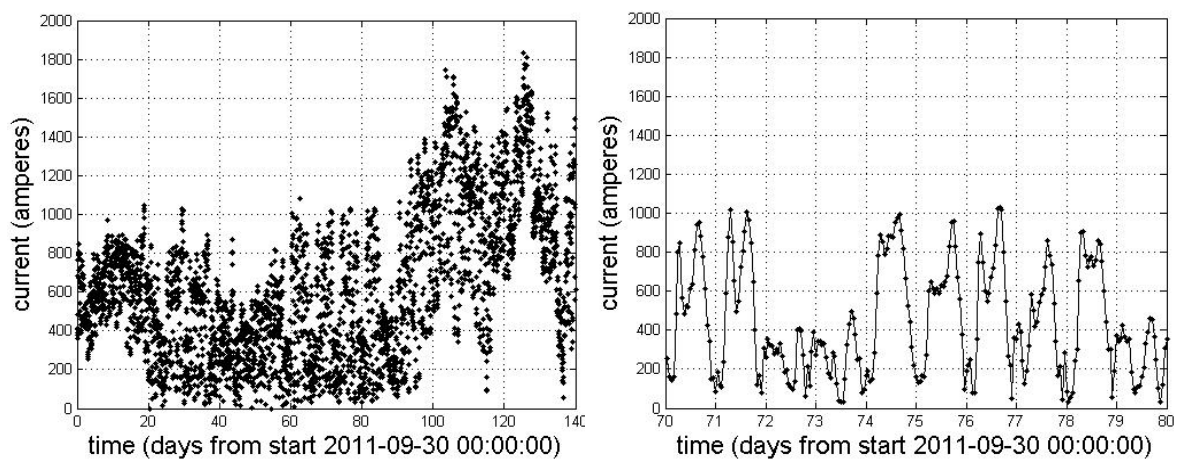

Note: The graph to the right represents only 10 days of the graph to the left.

In order to make a comparison to the linear models presented in Livshitz et al. (2005) and Chudnovsky (2007), the following linear model for the influence of the temperature on the current has been tried:

$$
T_{2}=b_{0}+b_{1} \cdot T_{1}+k_{1} \cdot I
$$

This model gives the results in Table 2. The biggest variation is in the parameter $k_{1}$, whose standard deviation divided by its mean is 0.350 . The same measure of variation gives 0.0175 for $b_{1}$ and -0.0390 for $b_{0}$.

Table 2 Parameters and $R^{2}$ estimated according to formula (3)

\begin{tabular}{lcccc}
\hline Sensor & $b_{0}\left({ }^{\circ} \mathrm{C}\right)$ & $b_{1}$ & $k_{1}\left({ }^{\circ} \mathrm{C} / \mathrm{kA}\right)$ & $R^{2}$ \\
\hline Phase 1 right & -9.20 & 0.929 & 3.87 & 0.974 \\
Phase 1 centre & -9.39 & 0.941 & 3.30 & 0.915 \\
Phase 1 left & -9.11 & 0.918 & 4.86 & 0.963 \\
Phase 2 right & -9.18 & 0.912 & 4.14 & 0.971 \\
Phase 2 centre & -8.19 & 0.918 & 2.43 & 0.945 \\
Phase 2 left & -8.96 & 0.929 & 3.46 & 0.964 \\
Phase 3 right & -8.73 & 0.951 & 2.01 & 0.929 \\
Phase 3 centre & -9.08 & 0.941 & 2.39 & 0.951 \\
Phase 3 left & -9.12 & 0.900 & 5.88 & 0.955 \\
\hline
\end{tabular}

\subsection{A quadratic model}

Since the dots in the scatter plot in Figure 13 could be overlapping, it is valuable to study the two-dimensional histogram in Figure 14. The histogram is also normalised for each interval of values of the current and plotted in colour, so the most common temperature residual is the reddest (the brightest in black-and-white). The curve following the brightest spots looks quadratic, which corresponds to the results in Chudnovsky et al. (2008). 
Figure 13 A scatter plot of temperature residuals (phase 1 centre against reference) versus current with a linear curve fitting the residuals

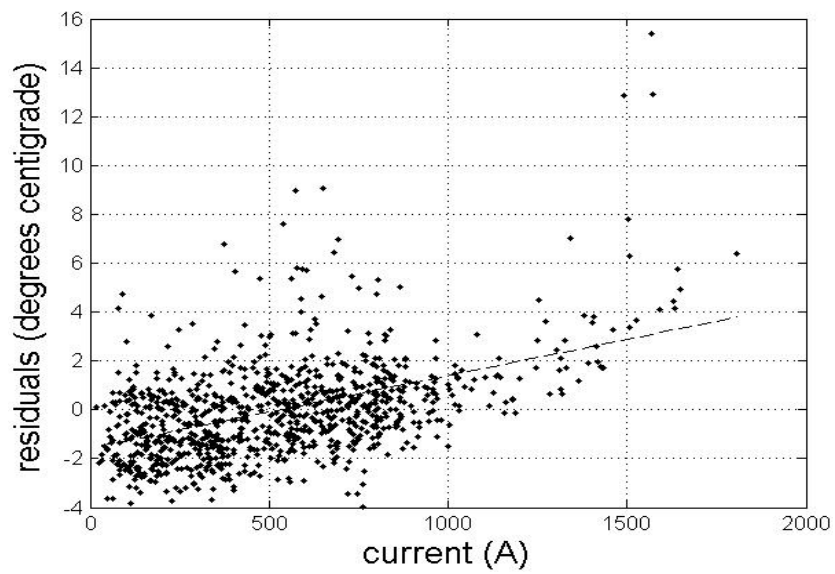

Figure 14 A normalised histogram of the temperature residuals (phase 1 centre against reference) versus current (see online version for colours)

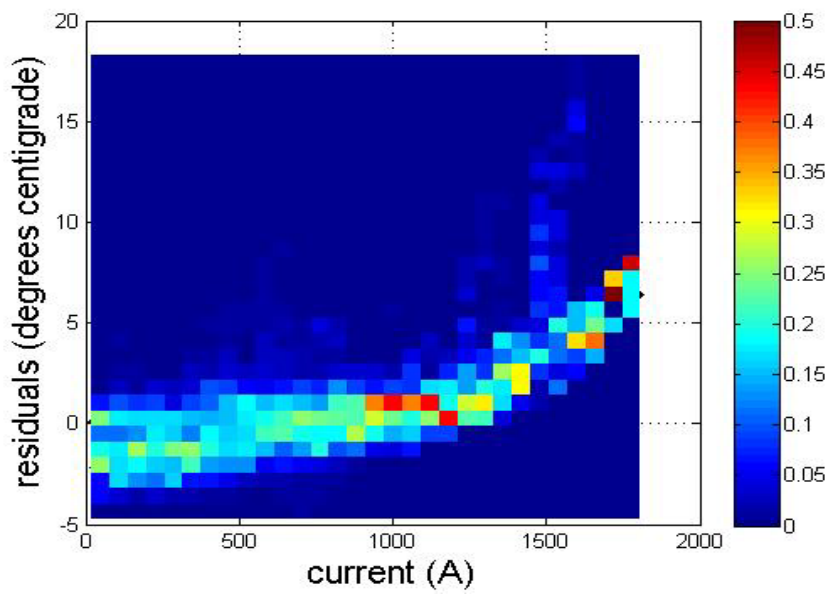

Note: A redder (brighter) square shows that there are more residuals for the interval of the current at this temperature interval.

According to Joule's law, $P=R \cdot I^{2}$ where $P$ is the power, $R$ the resistance and $I$ the current, the power losses increase with current and so does the temperature. Hence a regression is made to get the parameters $b_{0}, b_{1}$ and $k_{2}$ in the equation:

$$
T_{2}=b_{0}+b_{1} \cdot T_{2}+k_{2} \cdot I^{2}
$$

Table 3 shows the parameters $b_{0}, b_{1}$ and $k_{2}$ with the different sensors on the same disconnector as in the preceding section as $T_{2}$ and the reference sensor as $T_{1}$. Figure 15 shows the result of the regression. The biggest variation is in the parameter $k_{2}$, whose 
standard deviation divided by its mean is 0.338 . The same measure of variation gives 0.0194 for $b_{1}$ and -0.0497 for $b_{0}$.

Table 3 Parameters and $R^{2}$ estimated according to formula (4)

\begin{tabular}{lcccc}
\hline Sensor & $b_{0}\left({ }^{\circ} \mathrm{C}\right)$ & $b_{1}$ & $k_{2}\left({ }^{\circ} \mathrm{C} / \mathrm{k} \mathrm{A}^{2}\right)$ & $R^{2}$ \\
\hline Phase 1 right & -8.28 & 0.937 & 2.78 & 0.976 \\
Phase 1 centre & -8.95 & 0.971 & 2.72 & 0.922 \\
Phase 1 left & -7.96 & 0.929 & 3.43 & 0.965 \\
Phase 2 right & -8.23 & 0.921 & 2.97 & 0.974 \\
Phase 2 centre & -7.59 & 0.923 & 1.66 & 0.946 \\
Phase 2 left & -8.14 & 0.937 & 2.45 & 0.966 \\
Phase 3 right & -8.16 & 0.953 & 1.61 & 0.929 \\
Phase 3 centre & -8.50 & 0.946 & 1.66 & 0.951 \\
Phase 3 left & -7.72 & 0.912 & 4.21 & 0.960 \\
\hline
\end{tabular}

Figure 15 A scatter plot of temperature residuals (phase 1 centre against reference) versus current with a linear curve (dashed) and a quadratic curve (solid) fitting the residuals

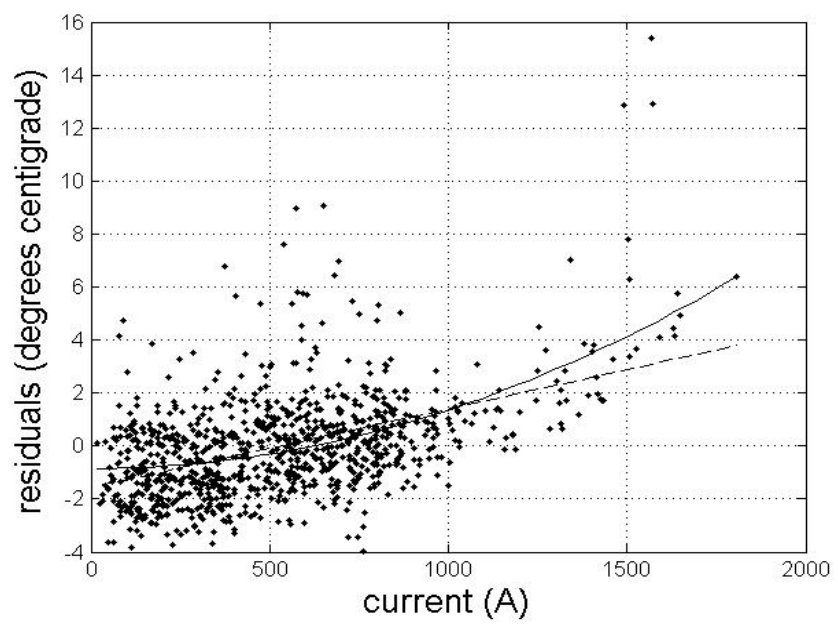

\section{Discussion}

When comparing the offset for the different models, $b_{0}$, it can be seen that it decreases when going from the basic to the linear model and then it increases slightly when going to the quadratic model, as illustrated in Figure 16. In the basic model, $b_{0}$ will be higher since it will be the mean for all currents, whereas in the linear model the temperature rise increases from a lower level with the current. The increase of the parameter $b_{0}$ in the quadratic model is due to the fact that the lower currents will give relatively lower increase of the temperature. 
Figure 16 The parameter $b_{0}$ for the basic model (dotted line), the linear model (dashed line) and the quadratic model (solid line)

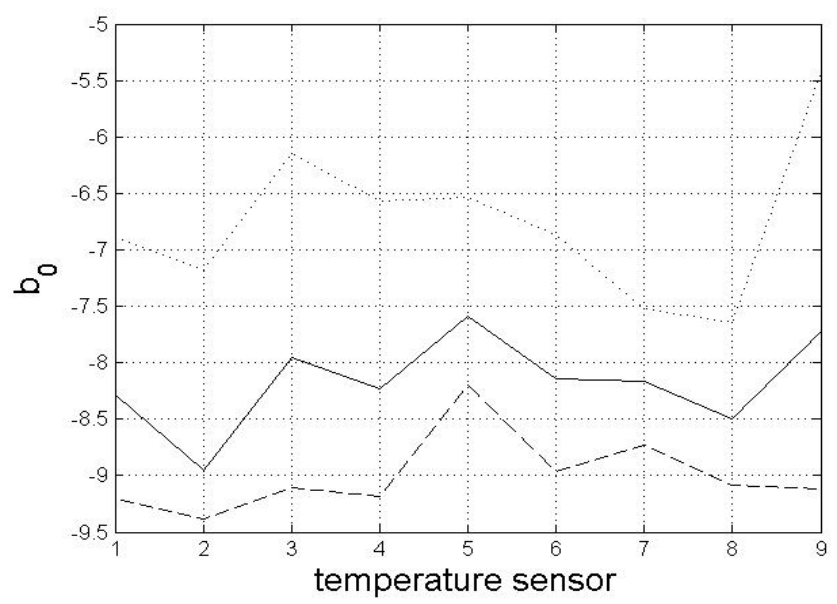

Note: The number of the sensor is the same as the row in the tables.

Figure 17 The parameter $k_{1}$ for the linear model (dashed line) and $k_{2}$ for the quadratic model (solid line)

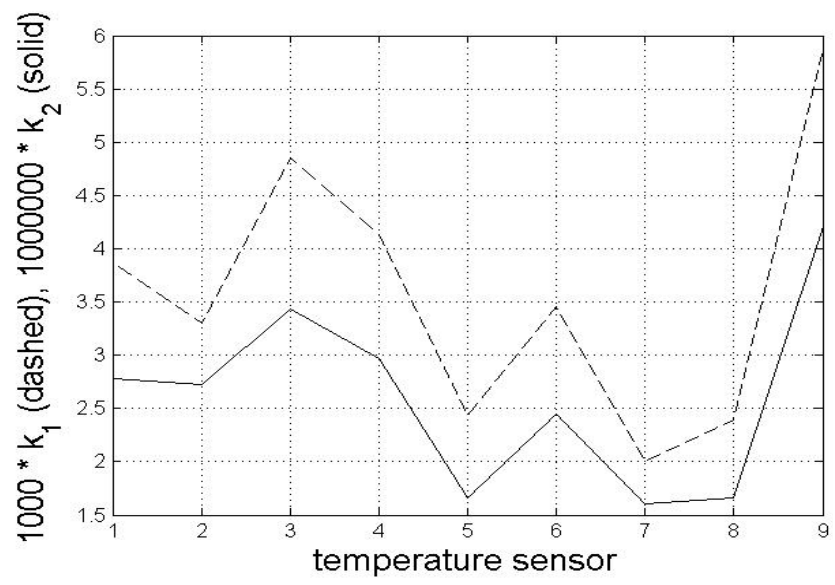

Note: The number of the sensor is the same as the row in the tables.

The parameter $b_{1}$ is about the same for all models, just below 1 , which is expected, since a change of the outdoor temperature should be followed by a similar change of the contact temperature. There is a significant difference in the variation of the parameters between the basic and the linear model. In the linear model the parameter $k_{1}$ has clearly most variation among the three parameters and the variation of $b_{0}$ decreases when going from the basic to the linear. It is interesting to note in Figure 17 that $k_{1}$ and $k_{2}$ show a similar pattern. For example, the highest values are seen for phase 3 left followed by phase 1 left and phase 2 right. The parameters $k_{1}$ and $k_{2}$ can be regarded as a measure of the contact condition and they may be used instead of the contact resistance. Since the 
parameters are ratio scales, they can be used to rank disconnector contacts of a population. Then it is possible to decide which disconnector should be maintained first, even though the remaining life cannot be calculated. This is a first step of condition-based maintenance.

The degree of explanation, $R^{2}$, has increased from between 0.887 and 0.954 for the basic model to between 0.922 and 0.976 for the quadratic model. The different $R^{2}$-values are shown in Figure 18 for the three models and the nine contact sensors regressed against the reference sensor. The quadratic model is the best and the linear model the second best. A straight-forward model with the outdoor temperature and the current can explain a great amount of the variation of the temperature of the contacts with one parameter that depends on the contact. It is also possible to test with different exponents of the current $I$. Then 2.0 is the best exponent for nearly all of the tested sensors. Some sensors have a difference in the exponent of about \pm 0.3 . Thus, a quadratic formula is reasonable.

Figure $18 R^{2}$ for the different contact sensors when regressed against the reference sensor

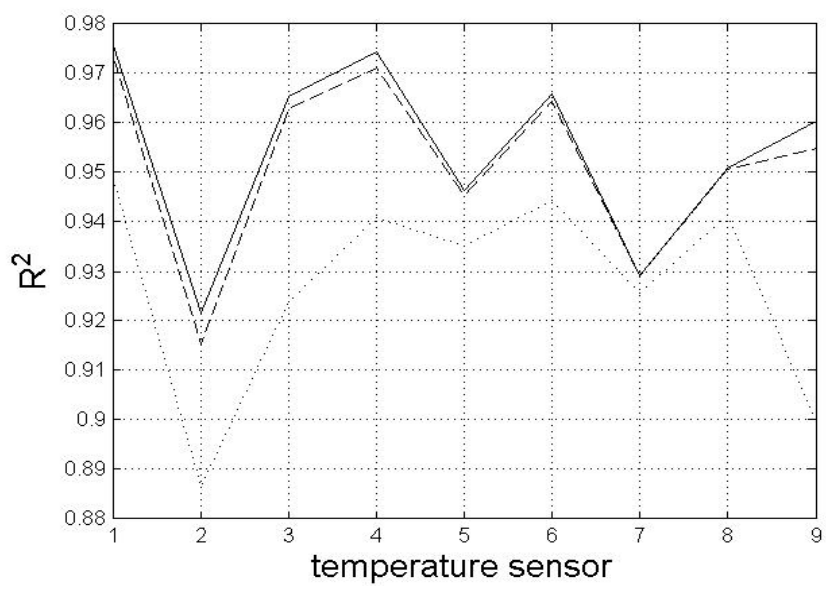

Notes: The number of the sensor is the same as the row in the tables. The dotted line corresponds to formula (1), the dashed line to formula (3) and the solid line to formula (4).

The difference in $R^{2}$ between the linear and the quadratic model is small. This is due to the overwhelming majority of small currents, less than 1,000 amperes, well below the maximum permitted current, 3,150 amperes. With a higher current there is a significant difference in the result. Figure 15 shows that for currents greater than 1,500 amperes (the right-most part of the graph), the quadratic model (the solid curve) fits the data better than the linear model (the dashed line). Another advantage is that the quadratic model corresponds to Joule's law.

In comparison to Zhang et al. (2012), Huang et al. (2011) and Shi et al. (2002), we have provided a method to interpret the data. Huda and Taib (2013) presented a more complex method, as it requires a more expensive sensor (thermographic camera) and complicated algorithms, even though the regions of interest must be selected manually. Furthermore, Huda and Taib (2013) do not consider the current, so a faulty condition 
with a low current might not be detected. Past measurements are not taken into account and the repeatability of thermography is a difficulty when interpreting a time series.

We have applied both the linear and the quadratic model presented in Chudnovsky et al. (2008) to disconnectors when they studied circuit breakers. The result is the same: the quadratic model is better. Muhr et al. (2006) claim that the exponent of the current is between 1.5 and 1.9, whereas our measurements give an exponent of 2.0 with a total variation of \pm 0.3 , which fits with Joule's law.

Our $R^{2}$-values for the different models, which shows how well the model explains the data, are lower than those presented in Chudnovsky et al. (2008, Table 1). They got really high values, 0.9847 and 0.9986 for the lowest values in the linear model and in the quadratic model, respectively, whereas we get $R^{2}$-values between 0.91 and 0.98 . A plausible reason for this is that their sensors are placed in closed compartments in equipment placed indoors. So, unlike our apparatus, there could not be any temperature changes due to the effect of solar heating or wind. Also the studied equipment, circuit breakers, has longer time constants.

\section{Conclusions and future work}

In this paper we have studied disconnectors and presented a measuring system, which can estimate the condition of the contacts. The system uses IR sensors fixed at the contacts to measure their temperature continuously. We have covered the first two steps of the reliability chain for disconnectors. The first step, measurements, is working with some problems connected to the new measurement technology, and we have found a method of condition estimation, which is the next step. With a straight-forward regression on the ambient temperature and the current, it is possible to rank the contacts in a disconnector population. This can lead to improved maintenance decisions and corresponds to a simplified version of the later steps in the reliability chain. By using extrapolation it can be investigated if it is likely that a disconnector will exceed temperature fault criteria at nameplate rating currents. Hence, it becomes possible to indicate faulty disconnectors before critical events take place. In addition disconnection of healthy components can be avoided, resulting in a total system performance improvement.

This study shows that the models applied to indoor circuit breakers in Chudnovsky et al. (2008) can also can be used for disconnectors, in a more exposed environment, outdoors. Their two models, linear and quadratic, have been tried and the quadratic model fits the data better. As expected, the temperature rise is proportional to the transferred power.

This paper studies one kind of disconnector, with two different kinds of contacts, one in the centre and two at the sides. The different contacts will be compared in a coming study, since they are constructed in different ways and have different thermal characteristics. Another future task is to understand how the parameters determined by the regression change with time. Both Braunovic et al. (2005) and Korendo and Florkowski (2001) have provided methods to analyse time series of the contact resistance. The current measurement period may be too short in order to observe changes in one disconnector. Hence, it is necessary to compare the conditions of the six disconnectors, since they are from three different decades. Another thing to be developed is a criterion for detecting failures. The influence of wind and solar radiation should also 
be estimated and possibly included in the model, using, for example, the study by Muhr et al. (2006).

\section{Acknowledgements}

The research is funded by SweGRIDS, the Swedish Centre for Smart Grids and Energy Storage. We thank the reviewers and Carl Johan Wallnerström and Sajeesh Babu at the School of Electrical Engineering at KTH Royal Institute of Technology and for valuable comments.

\section{References}

Andersson, G., Donalek, P., Farmer, R., Hatziargyriou, N., Kamwa, I., Kundur, P., Martins, N., Paserba, J., Pourbeik, P., Sanchez-Gasca, J., Schulz, R., Stankovic, A., Taylor, C. and Vittal, V. (2005) 'Causes of the 2003 major grid blackouts in North America and Europe, and recommended means to improve system dynamic performance', IEEE Transactions on Power Systems, Vol. 20, No. 4, pp.1922-1928.

Bagavathiappan, S., Lahiri, B., Saravanan, T., Philip, J. and Jayakumar, T. (2013) 'Infrared thermography for condition monitoring - a review', Infrared Phys. Technol., September, Vol. 60, No. 21, pp.35-55 (Netherlands).

Braunovic, M., Izmailov, V. and Novoselova, M. (2005) 'A model for life time evaluation of closed electrical contacts', Proceedings of the Annual Holm Conference on Electrical Contacts, pp.217-223, Chicago, IL, USA.

Braunovic, M., Izmailov, V., Naumov, A. and Novoselova, M. (2009) 'Estimation of remaining lifetime of power connections using infrared thermography', Proceedings of the 55th IEEE Holm Conference on Electrical Contacts, pp.234-239.

Chudnovsky, B., Livshitz, A. and Chudnovsky, B. (2008) 'Thermal model of electrical contacts based on experimental data', 2008 IEEE Holm Conference on Electrical Contacts, pp.205-211, Piscataway, NJ, USA.

Chudnovsky, B.H. (2007) 'Electrical contacts condition diagnostics based on wireless temperature monitoring of energized equipment', Electrical Contacts, Proceedings of the Annual Holm Conference on Electrical Contacts, pp.73-80, Montreal, QC, Canada.

Cramér, H. (1945) Mathematical Methods of Statistics, Almqvist \& Wiksell, Uppsala.

Eriksson, L. (2005) Han stoppar elavbrott (He Prevents Power Outages) Ny Teknik, (3) Published on the 25th of January in Swedish [online] http://www.nyteknik.se/nyheter/it_telekom/allmant/article240223.ece.

Hogg, R.V. and Tanis, E.A. (1993) Probability and Statistical Inference, 4th ed., Macmillan, New York.

Holm, R. (1967) Electric Contacts, Theory and Applications, 4th ed., Springer-Verlag, Berlin.

Huang, X., Huang, B. and Wang, H. (2011) 'Design of compositive on-line monitoring and fault diagnosis system for high-voltage switch cabinet', International Conference on Electrical and Control Engineering, ICECE 2011, pp.156-159, Yichang, China.

Huda, A.N. and Taib, S. (2013) 'Application of infrared thermography for predictive/preventive maintenance of thermal defect in electrical equipment', Applied Thermal Engineering, Vol. 61, No. 2, pp.220-227.

Jadin, M.S. and Taib, S. (2012) 'Recent progress in diagnosing the reliability of electrical equipment by using infrared thermography', Infrared Physics and Technology, Vol. 55, No. 4, pp.236-245. 
Korendo, Z. and Florkowski, M. (2001) 'Thermography-based diagnostics of power equipment', Power Engineering Journal, Vol. 15, No. 1, pp.33-42.

Lindquist, T. (2011) Wireless sensors vs thermography - comparing accuracy and applications', SC A3 Colloquium, No. A3-202 2011, Vienna, CIGRÉ.

Lindquist, T. and Bertling, L. (2007) 'A method for calculating disconnector contact availability as a function of thermography inspection intervals and load current', Cigré Symposium on System Development and Asset Management under Restructuring, Osaka.

Lindquist, T., Bertling, L. and Eriksson, R. (2005) 'Estimation of disconnector contact condition for modelling the effect of maintenance and ageing', IEEE Russia Power Tech., 27-30 June 2005, St. Petersburg, pp.1-7.

Livshitz, A., Chudnovsky, B.H., Bukengolts, B. and Chudnovsky, B.A. (2005) 'On-line temperature monitoring of power distribution equipment', Record of Conference Papers Annual Petroleum and Chemical Industry Conference, pp.223-231, Denver, CO, USA.

Muhr, M., Pack, S., Jaufer, S. and Lugschitz, H. (2006) 'Thermography of aged contacts of high voltage equipment', Elektrotechnik und Informationstechnik, Vol. 123, No. 12, pp.537-543.

Sen, A. and Srivastava, M. (1990) Regression Analysis. Theory, Methods, and Applications, Springer-Verlag, New York.

Shi, Z., Meng, Y., Yang, W., Ding, D., Jia, S., Rong, M. and Cheng, Y. (2002) 'On-line condition monitoring system of medium-voltage switchgear', Proceedings of the IEEE Power Engineering Society Transmission and Distribution Conference, Vol. 3, pp.2290-2294, Yokahama, Japan.

Stanisic, Z. (2011) 'Method for static and dynamic resistance measurements of HV circuit breaker', in IEEE PES Innovative Smart Grid Technologies Conference Europe, Manchester, UK.

Svenska kraftnät (2013) 'Perspektivplan 2025 - en utvecklingsplan för det svenska stamnätet (perspective plan 2025 - a plan for the development of the Swedish electrical transmission system)' [online] http://www.svk.se/rapporter (accessed 29 November 2013).

Svenska kraftnät (2014) 'Stamnätet (the transmission grid)' [online] http://www.svk.se/Om-oss/Varverksamhet/Stamnatet/ (accessed 1 April 2014).

Zhang, J., Gao, Y. and Hao, P. (2012) 'Research on on-line high-voltage switch contact temperature rise monitoring system based on WSN', Advanced Materials Research, Vol. 382, pp.210-214, Beijing, China. 